\title{
APLICAÇÃO DA CURVA ABC E SEU EFEITO NA ACURACIDADE DO ESTOQUE EM UMA EMPRESA DE REVENDA DE KIT DE EMBREAGEM NA REGIÃO DA ZONA DA MATA MINEIRA
}

\author{
APPLICATION OF THE ABC CURVE AND ITS EFFECT ON STOCK ACCURACY IN A \\ CLUTCH KIT RESALE COMPANY IN THE MATA MINEIRA ZONE REGION
}
Leonardo Chahim Pereira', Bruno Foureaux Fabbis ${ }^{2}$, Mário Cesar Fialho de Oliveira ${ }^{3}$, Patrícia Werneck Silva de Oliveira ${ }^{4}, \&$ Sanderson Rocha de Abreu ${ }^{5}$
12345 Departamento de Engenharias e Tecnologia da FIC - Faculdades Integradas de Cataguases do Grupo Unis, R. Romualdo Menezes, 701, Bairro Menezes, CEP 36773-084, Cataguases.
1* chahim@outlook.com.br ${ }^{2}$ brunoffabis@gmail.com ${ }^{3}$ mc-mariocesar@hotmail.com ${ }^{4}$ paticso@yahoo.com.br 5 sanderson.abreu@unis.edu.br

\section{ARTIGO INFO.}

Recebido em: 14.10.2020

Aprovado em: 12.11.2020

Disponibilizado em: $\mathbf{1 8 . 1 1 . 2 0 2 0}$

\section{Palavras-chave:}

Classificação ABC; gestão de estoque; acuracidade; plano de contagem; inventário cíclico.

\section{KEYWORDS:}

ABC classification, inventory management, accuracy, counting plan; cyclical inventory.

*Autor Correspondente: Pereira, L. C.

\section{RESUMO}

Neste estudo evidencia-se o efeito da classificação $\mathrm{ABC}$ na gestão de estoque, mais especificamente na acuracidade do estoque. Para desenvolvimento do presente estudo inicialmente fez-se uma revisão bibliográfica acerca de temas centrais para desenvolvimento da temática e posteriormente realizou-se o estudo prático através de visitas em uma empresa revendedora de KIT de embreagem, localizada na região da Zona da Mata Mineira, com o intuito de viabilizar a gestão de estoques a partir da aplicação da Curva ABC, que por sua vez possibilitou uma elaboração de um plano de contagens físico do inventário rotativo por meio de períodos e ciclos para cada classe A, B e C, buscando assim uma melhor acurácia no estoque da empresa. Foi possível concluir que a gestão de estoque com a aplicação do da curva ABC tem efeito positivo sobre o índice de acuracidade dos estoques, porque auxilia na estruturação do plano de contagem e na otimização das tomadas de decisões, bem como no aumento do índice de acurácia do estoque, onde na empresa estudada a acurácia dos itens aumentou de $64,22 \%$ para $97,59 \%$, conseguindo atingir um percentual de divergência melhor do que o estabelecido pela empresa.

\begin{abstract}
This study shows the effect of the ABC classification on inventory management, more specifically on stock accuracy. For the development of the present study, a bibliographic review was made about the central themes for the development of the theme and later the practical study was carried out through visits to a clutch KIT reseller company, located in the Zona da Mata Mineira region, with in order to make inventory management viable by applying the ABC Curve, which in turn made it possible to draw up a physical counting plan for the rotating inventory through periods and cycles for each class A, B and C, thus seeking a better accuracy in the company's stock. It was possible to conclude that the stock management with the application of the $\mathrm{ABC}$ curve has a positive effect on the inventory accuracy index, because it helps in structuring the counting plan and in the optimization of decision making, as well as in increasing the accuracy index. Of the stock, where in the company studied the accuracy of the items increased from $64.22 \%$ to $97.59 \%$, achieving a better divergence percentage than that established by the company.
\end{abstract}


Citação (APA): Pereira, L. C., Fabbis, B. F., Oliveira, M. C. F. de, Oliveira, P. W. S. de, \& Abreu, S. R. de. (2020). Aplicação da curva abc e seu efeito na acuracidade do estoque em uma empresa de revenda de kit de embreagem na região da zona da mata mineira. Brazilian Journal of Production Engineering, 6(7), 58-67.

\section{INTRODUÇÃO}

Segundo Moreira, Silva e Ebias (2019), com os avanços das tecnologias e o aumento da competitividade no cenário atual, o desafio das instituições é garantir a satisfação dos seus clientes, a qualidade total nos seus produtos e serviços e a redução dos custos. Dessa forma, se faz necessário obter conhecimentos de métodos de gerenciamento e técnicas para otimização dos seus processos.

Para Silva (2019), é imprescindível o conhecimento das características e dinâmicas dos estoques, pois em ambientes incertos os mesmos elevam o grau de segurança. Nesse sentido, é sempre importante manter estoques para atendimento de demandas da programação de produção e/ou demandas de clientes. Dessa forma, os estoques são uma garantia diante do imprevisto. Esse é o grande dilema dos estoques: apresentam riscos e custos inerentes, enquanto auxiliam a conciliar fornecimento e demanda. Assim, pode-se afirmar que os estoques só existem devido à falta de sincronismo entre demanda e fornecimento.

Para Accioly, Ayres e Sucupira (2015), as empresas comerciais e industriais são mais dependentes da gestão de estoques quando comparadas às que atuam no setor de serviços, devido ao fato de possuírem, representativos volumes atrelados aos seus inventários, que quando traduzidos em dinheiro e comparados com o valor necessário para impulsionar as atividades. Dessa forma, a gestão de estoque deve estar atrelada com o planejamento estratégico da empresa, devido a necessidade de manter a mesma competitiva no mercado, onde as mudanças são frequentes.

Uma gestão de estoque eficaz contribui para o equilíbrio econômico financeiro da organização, auxilia no planejamento financeiro anual, controla o inventário e potencialmente melhora a lucratividade. Sendo assim, torna-se necessário o extremo cuidado com as informações de estoque, para que os saldos em estoque demonstrados nos sistemas informatizados mantenham perfeita sintonia com os saldos físicos, existentes nos depósitos. Uma adequada gestão de estoque baseada na classificação $\mathrm{ABC}$, que seja capaz de estabelecer parâmetros de gerenciamento da acuracidade do estoque, tende a ser adequada à organização, pois indica para cada classe um período de contagem para a acuracidade do estoque.

O presente estudo tem como principal objetivo averiguar se a utilização da Curva $A B C$ traz melhores resultados sobre a gestão de estoque em uma empresa de pequeno porte de revenda de kit de embreagem. Buscou-se a resposta para a seguinte questão de pesquisa: $\mathrm{O}$ uso da ferramenta de Classificação ABC tem efeito sobre o Índice de Acuracidade do Estoque?

No intuito de alcançar o objetivo geral, foram definidos os seguintes objetivos específicos:

(i) Coletar os valores unitários e a quantidade consumida de todos os tens revendidos pela empresa em 2019;

(ii) Determinar o investimento anual (preço unitário x consumo anual);

(iii) Ordenar os itens do maior para o menor investimento;

(iv) Calcular a porcentagem que cada item representa no investimento total e a porcentagem acumulada;

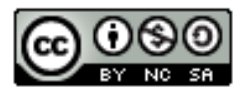


Citação (APA): Pereira, L. C., Fabbis, B. F., Oliveira, M. C. F. de, Oliveira, P. W. S. de, \& Abreu, S. R. de. (2020). Aplicação da curva abc e seu efeito na acuracidade do estoque em uma empresa de revenda de kit de embreagem na região da zona da mata mineira. Brazilian Journal of Production Engineering, 6(7), 58-67.

(v) Realizar uma classificação dos materiais, através da curva ABC, de acordo com sua importância e valor para a empresa, determinando o produto mais crítico com critérios embasados em literatura;

(vi) Coletar a quantidade de requisições para cada item e determinar o percentual acumulado;

(vii) Determinar um plano de contagem de inventário cíclico e aplicar por 3 meses na empresa;

(viii) Determinar acurácia após aplicação do plano de contagem e compara-la com a de 2019.

Este artigo está estruturado da seguinte forma. A Seção 1 introduz as referências teóricas. $\mathrm{Na}$ seção 2 é descrita a metodologia da pesquisa. A seção 4 apresentam-se resultados e discussões. A Seção 5 traz conclusões, contribuições, limitações e possibilidades futuras de pesquisa.

\section{REVISÃO DE LITERATURA}

\subsection{ESTOQUE}

Para Candido e Marcos (2019), estoque é definido como o acumulo armazenado de materiais em um sistema de transformação e, algumas vezes, para apresentar qualquer recurso armazenado. Ainda segundo Candido e Marcos (2019) o estoque existe porque não há um sincronismo entre demanda e fornecimento. Se o fornecimento de qualquer produto ocorresse na hora em que fosse demandado o mesmo nunca seria estocado.

Segundo Corrêa e Corrêa (2017), os estoques estão entre as maiores preocupações dos gerentes de operações e dos gestores financeiros. Na perspectiva operacional, baixos estoques significam indisponibilidade de atendimento ao cliente, enquanto na visão financeira, estoques altos significam dinheiro parado e, consequentemente, maiores custos.

\subsection{GESTÃO DE ESTOQUE}

Segundo Santos (2017 apud Souza et al., 2018) a gestão de estoque é a forma de controlar os recursos ociosos com importância financeira que são designados ao abastecimento das necessidades futuras do material em uma instituição. $O$ gerenciamento de estoque começou por necessidade das empresas em controlar todo o fluxo de materiais como a quantidade, tempo, entre outros.

Para Silva (2019) a gestão de estoque tem uma função integradora, devido ao fato de envolver diversas áreas dentro de uma instituição (produção, marketing, vendas, logística, compras, assistência técnica, entre outras). Dessa forma, as decisões da gestão de estoques não devem se limitar aos níveis intermediários na hierarquia da instituição, pois, sendo assim, ela perderia a visão estratégica, podendo comprometer os objetivos estratégicos da instituição. Portanto, a gestão de estoques deve ser realizada sob orientação das diretrizes estratégicas da instituição, de modo que a ótica da empresa prevalece sobre os interesses individuais dos departamentos.

\subsection{INVENTÁRIO}

Segundo Silva (2019), o inventário físico dos estoques é o balanço dos itens disponíveis da instituição para serem comparados com os registros contábeis dos mesmos. Os inventários são levantamentos de itens que efetivamente hão em estoque, frente ao quanto deveria haver, com base nos registros de contabilidade. Eles podem ser (i) periódicos, sendo realizados em um

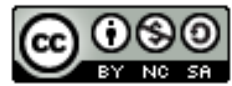


Citação (APA): Pereira, L. C., Fabbis, B. F., Oliveira, M. C. F. de, Oliveira, P. W. S. de, \& Abreu, S. R. de. (2020). Aplicação da curva abc e seu efeito na acuracidade do estoque em uma empresa de revenda de kit de embreagem na região da zona da mata mineira. Brazilian Journal of Production Engineering, 6(7), 58-67.

intervalo de tempo, podem ser feitos anualmente ou semestralmente; ou (ii) cíclicos, realizados gradualmente, sem necessidade de paradas das operações ou fechamento da empresa.

"Os sistemas de inventário são importantes ferramentas para manter a acuracidade dos estoques e dar confiabilidade aos números informados aos setores de suprimentos, vendas, programação e contabilidade" (Paoleschi, 2018).

\section{METODOLOGIA}

Inicialmente realizou-se uma revisão bibliográfica acerca de temas centrais para desenvolvimento da temática do trabalho, buscando-se fundamentação na literatura para cumprir com o objetivo do trabalho. Posteriormente, realizou-se o estudo prático, através de visitas a empresa para análise dos problemas apresentados e coleta de informações, com o intuito de viabilizar a gestão de estoques a partir da aplicação da Curva $\mathrm{ABC}$, que por sua vez possibilitou uma elaboração de um plano de contagens físico do inventário rotativo por meio de períodos e ciclos para cada classe, buscando assim uma melhor acurácia no estoque

Os dados foram coletados de uma empresa de pequeno porte, revendedora de Kit de Embreagem, localizada na região da Zona da Mata Mineira. A empresa objeto de análise relata vários problemas com seu estoque, não tendo atingido a meta de $95 \%$ de acuracidade de estoque estabelecida, devido à falta de controle no ato de recebimento, armazenagem e saída de produtos. Esses problemas são apresentados no momento do inventário dos estoques e consecutivamente baixo índice de acuracidade dos estoques.

$\mathrm{Na}$ análise dos dados foram consideradas as mercadorias registradas na empresa como Kit de Embreagem e suas vendas durante o ano de 2019, informações extraídas do software de gestão de estoque interno da empresa, responsável pelo controle de entrada e saída dos estoques. Para relacionar os produtos, separá-los de acordo com a importância e implementar a Curva ABC, utilizou-se recursos do programa Microsoft Office Excel. O período de tempo escolhido foi suficiente para análise dos dados e não muito longo para se observar influências sazonais de demanda.

\subsection{CLASSIFICAÇÃO ABC}

Segundo Paoleschi (2019), Curva ABC, ou Classificação ABC, é um método para classificação dos itens de maior importância que são, normalmente, em menor número. Tem como base o teorema do economista e sociólogo italiano Vilfredo Pareto (1848-1923). Na avaliação dos resultados da curva $\mathrm{ABC}$ percebe-se o nível da lucratividade, o grau de representação no faturamento e o giro dos itens no estoque.

Para Pozo (2010 apud Facchini, Silva, \& Leite, 2019) a Curva ABC possibilita a rápida tomada de decisão, sendo a classe A responsável em média, por $80 \%$ do valor monetário total e $20 \%$ dos itens; a classe B, responsável, em média, por $15 \%$ do valor monetário total e $30 \%$ dos itens; e a classe $\mathrm{C}$, responsável, em média, por $5 \%$ do valor monetário total e $50 \%$ dos itens.

Para elaboração da Curva ABC, com o objetivo de determinar a classificação dos itens de estoque da empresa, foram coletados os valores unitários e a demanda no ano de 2019 para todos os 44 itens revendidos pela empresa. Após a coleta, multiplicou-se o valor unitário de 
Citação (APA): Pereira, L. C., Fabbis, B. F., Oliveira, M. C. F. de, Oliveira, P. W. S. de, \& Abreu, S. R. de. (2020). Aplicação da curva abc e seu efeito na acuracidade do estoque em uma empresa de revenda de kit de embreagem na região da zona da mata mineira. Brazilian Journal of Production Engineering, 6(7), 58-67.

cada item pela demanda anual, obtendo-se assim a receita gerada por cada item. As receitas foram somadas para se obter a receita total. A seguir, obtêm-se a porcentagem de cada receita sobre o somatório total e, por fim, os itens são classificados na ordem decrescente pelo percentual acumulado, determinando a classe de cada item, com base na literatura informada.

\subsection{PLANO DE CONTAGEM}

Segundo Gasnier (2002) na construção do plano de contagens são programadas as contagens físicas do inventário rotativo, através de períodos e ciclos. O período corresponde ao tempo necessário para se contar todos os itens de estoque pelo menos uma vez. Os ciclos são as contagens realizadas dentro do período, podendo haver um ou mais ciclos dentro de um determinado período, ou seja, são as frequências das contagens.

Dessa forma, para a elaboração do plano de contagem, determinou-se a frequência de contagem em função da curva ABC, conforme indicado por Gasnier (2002). Gasnier cita que para cada classe é determinada uma frequência de contagem que, por sua vez ,pode ser alterada dependendo da avaliação do gestor de materiais e demais envolvidos, porém neste estudo seguimos exatamente o informado por Gasnier para realização do plano de contagem.

Aplicou-a para os 44 itens classificados na curva $\mathrm{ABC}$, durante um período de 3 meses ou 12 semanas, compreendendo o intervalo de 10/02/2020 até 10/05/2020.

Dessa forma, para a classe A, para a qual foram determinados 4 ciclos por período, realizou-se a contagem de 3 itens na primeira semana, 3 itens na segunda semana e 2 itens na terceira semana, totalizando assim 8 itens, repetindo essa contagem 4 vezes por período. Para a classe $\mathrm{B}$, para a qual foram determinados 2 ciclos por período, realizou-se a contagem de 3 itens a cada semana das cinco primeiras e 2 itens na sexta e última semana, totalizando assim 17, repetindo essa contagem 2 vezes por período. Para a classe $C$, na qual foi determinada 1 ciclo por período, realizou-se a contagem de 2 itens a cada semana das sete primeiras e 1 item a cada semanada das 5 últimas, totalizando assim 19.

\subsection{ACURACIDADE DE ESTOQUE}

Segundo Bertaglia (2003 apud Moreira, Silva, \& Ebias, 2019), a acurácia de estoque é um indicador essencial que demonstra a qualidade e a confiabilidade das informações armazenadas no sistema de controle, pois mede o nível de assertividade entre estoque físico e sistema.

Para Silva (2019) os desvios de estoque entre o físico e o contabilizado pelo sistema podem ser analisados através dos Índices de Acurácia dos Estoques, esse índice mostra o percentual dos estoques sem necessidade de ajuste, ou seja, mostra o grau de controle ou descontrole que a empresa tem de seus estoques, pode ser calculado pela equação 1:

$$
I A E=\frac{S F}{S S} \times 100
$$

Fonte: Bertaglia (2006 apud Moreira, Silva e Ebias, 2019)

Onde:

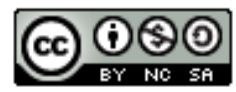



of Production Engineering, 6(7), 58-67.

(i) IAE: índice de acurácia de estoques;

(ii) SF: quantidade de saldo após contagem;

(iii)SS: quantidade de saldo verificada no sistema.

Para determinação da acurácia no ano de 2019 e após aplicação do plano de contagem, foi necessário contabilizar fisicamente o saldo dos 44 itens no estoque e depois soma-los, dividindo-os pelo somatório de saldos de cada item no sistema, encontrando a porcentagem, que é o índice de acurácia (IAE), conforme cálculo da IAE.

\section{RESULTADOS E DISCUSSÃO}

O principal fator a ser analisado nesse estudo foi o indicador de acuracidade dos estoques. Sabendo-se que o plano de contagem era realizado anualmente pela empresa, realizou-se o inventário físico no almoxarifado, referente ao ano de 2019, onde obteve-se com auxílio do cálculo do IAE, a acuracidade total dos 44 itens.

A empresa em estudo admite um percentual de divergência de 5\% na acuracidade total dos itens. Porém, com a análise dos saldos de inventário e dos cálculos realizados, observou-se que a acuracidade de 2019 foi de 64,22\%, obtendo-se assim um percentual de divergência de $35,78 \%$, ou seja, um percentual acima do estipulado. Segundo Gasnier (2002), a acurácia de saldo é um indicador gerencial que determina para a tecnologia de contagem periódica um índice de acerto de 90\%, ou seja, admite um percentual de divergência de 10\%. Dessa forma podemos concluir que, para a contagem periódica, a empresa utiliza um percentual mais rigoroso do que o informado por Gasnier (Figura 1).

Com o objetivo de melhorar a acurácia, realizamos a classificação dos itens, foi possível construir o gráfico da curva ABC.

Figura 1. Curva ABC

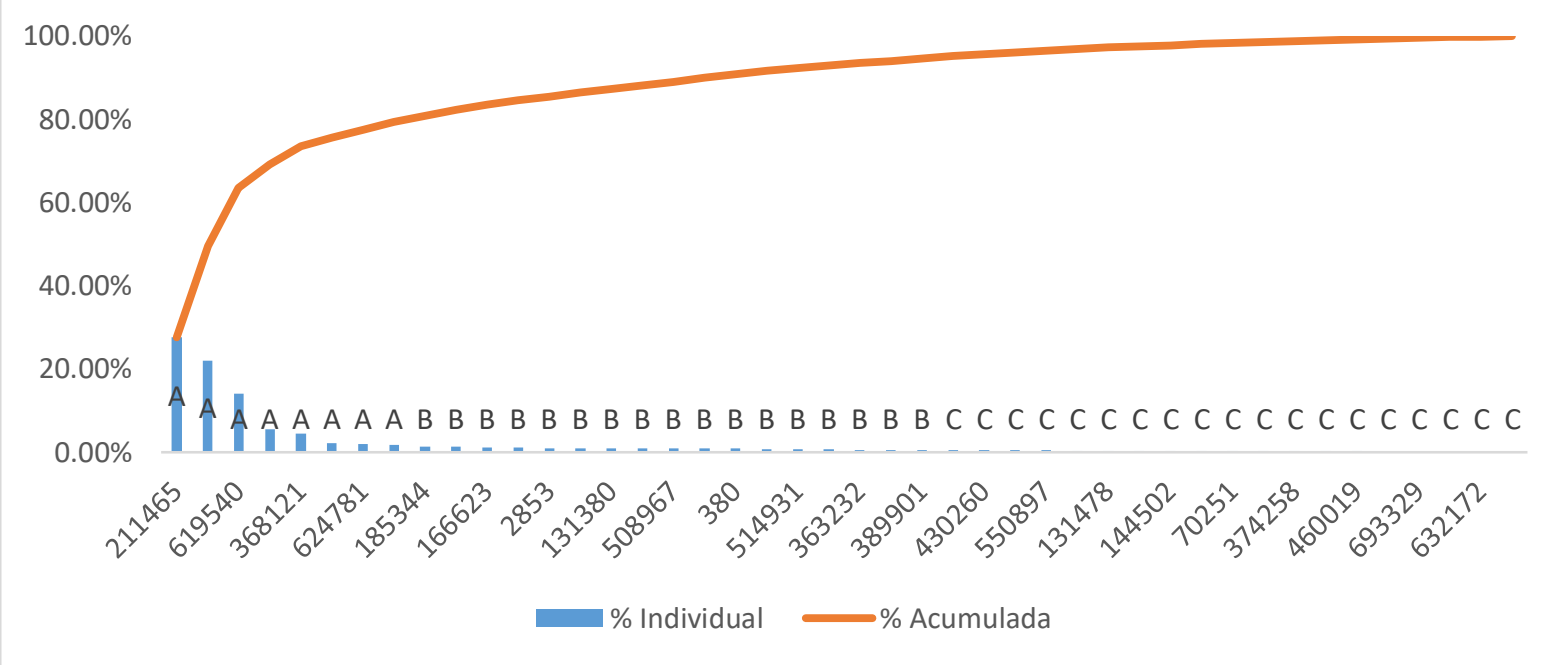

Fonte: Autores (2020).

Pode-se analisar que os itens são ordenados da esquerda para a direita, da maior para a menor porcentagem de receita que representam a porcentagem acumulada da receita é colocada em ordem decrescente. A curva de \% Acumulada sobe rapidamente devido aos primeiros itens 
Citação (APA): Pereira, L. C., Fabbis, B. F., Oliveira, M. C. F. de, Oliveira, P. W. S. de, \& Abreu, S. R. de. (2020). Aplicação da curva abc e seu efeito na acuracidade do estoque em uma empresa de revenda de kit de embreagem na região da zona da mata mineira. Brazilian Journal of Production Engineering, 6(7), 58-67.

representarem maior importância em termos de receitas geradas. Posteriormente, o crescimento torna-se mais lento, até atingir $100 \%$ que corresponde aos itens menos importantes. Dessa forma, por meio da Curva $\mathrm{ABC}$, foi possível elaborar um plano de contagem para cada classe.

O Quadro 1 apresenta o resumo das informações referente a Curva ABC dos estoques. Pode-se observar que $18 \%$ dos itens, ou seja, 8 dos 44 itens analisados, correspondem a 79,41\% dos investimentos da empresa. Estes são classificados como itens classe A. A classe B, que é intermediaria, possui 17 itens, que correspondem a 15,20\% do investimento. E por fim, a classe C corresponde a um investimento de 5,39\%, com um total de 19 itens, que por sua vez representa uma relevância pequena.

Quadro 1. Resumo do grau de importância da curva ABC

\begin{tabular}{|c|c|c|rr|c|}
\hline Classes & $\begin{array}{c}\text { Porcentagem } \\
\text { de itens }\end{array}$ & $\begin{array}{c}\text { Porcentagem } \\
\text { de Valor }\end{array}$ & Valor em Estoque & Importância \\
\hline $\mathrm{A}$ & $18 \%$ & $79,41 \%$ & $\mathrm{R} \$$ & $279.045,00$ & Grande \\
\hline $\mathrm{B}$ & $39 \%$ & $15,20 \%$ & $\mathrm{R} \$$ & $53.424,00$ & Intermédiaria \\
\hline $\mathrm{C}$ & $43 \%$ & $5,39 \%$ & $\mathrm{R} \$$ & $18.934,00$ & Pequena \\
\hline Total & $100 \%$ & $100 \%$ & $\mathrm{R} \$$ & $351.403,00$ & \\
\hline
\end{tabular}

Fonte: Autores (2020)

Em função da Curva ABC, estabelecemos o plano de contagem representado na FIGURA 2, que propõe um modelo de inventário cíclico, baseado na classificação dos itens. No plano de contagem podemos observar que para a classe $\mathrm{A}$, temos uma frequência de 4 ciclos por período, na classe $\mathrm{B}$ temos 2 ciclos por período e na classe $\mathrm{C}$ temos 1 ciclo por período.

Figura 2. Plano de contagem em função da curva ABC

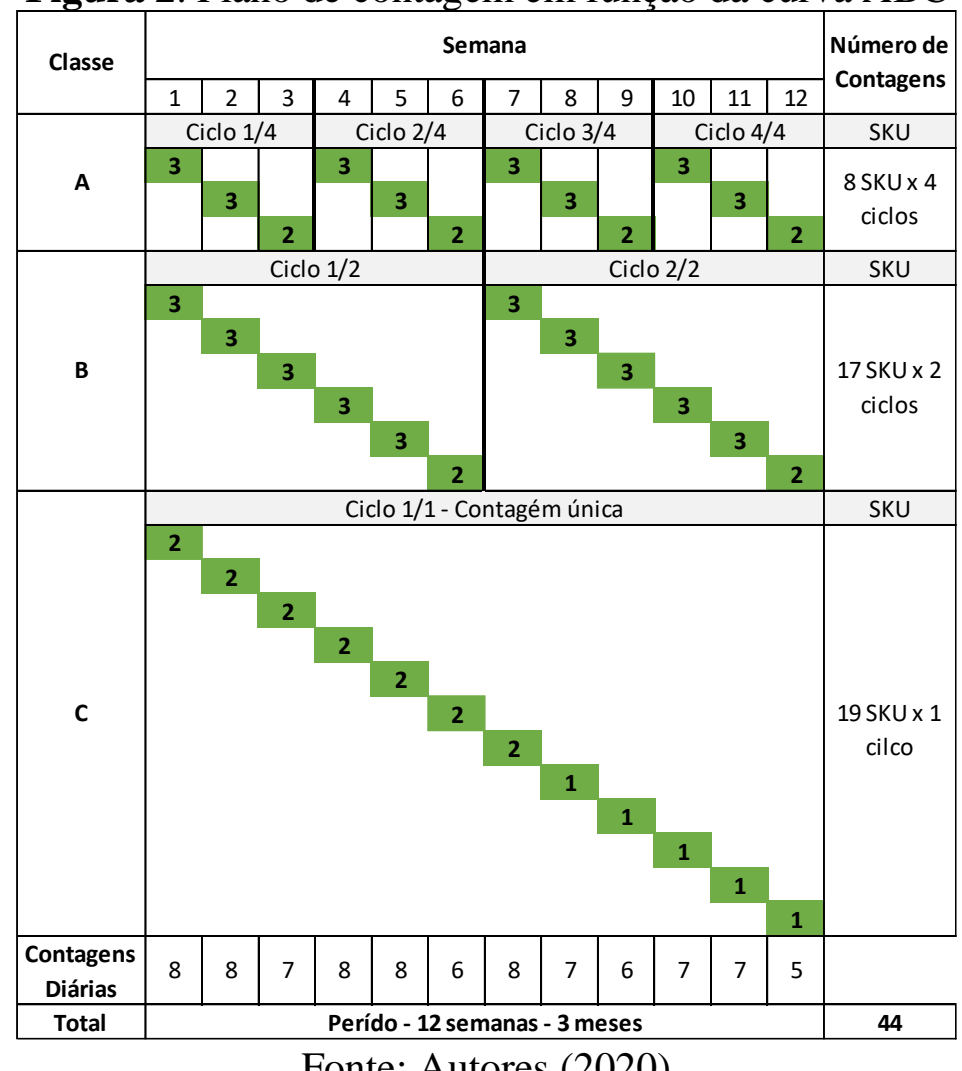

Fonte: Autores (2020) 

of Production Engineering, 6(7), 58-67.

Após a aplicação do plano de contagem de inventário cíclico, durante o período de 3 meses, foi possível estabelecer uma acuracidade de estoque de $97,59 \%$ e consequentemente obtemos uma divergência percentual de $2,41 \%$, conseguindo alcançar o percentual de divergência estipulado pela empresa que são $5 \%$.

A Figura 3 mostra uma representação gráfica da acurácia antes do plano de contagem totalizando $64,22 \%$ de acuracidade dos estoques e depois do plano de contagem totalizando 97,59\% e a meta estipulada pela empresa para acuracidade dos estoques que é $95 \%$.

Figura 3. Acurácia do estoque antes e após o plano de contagem

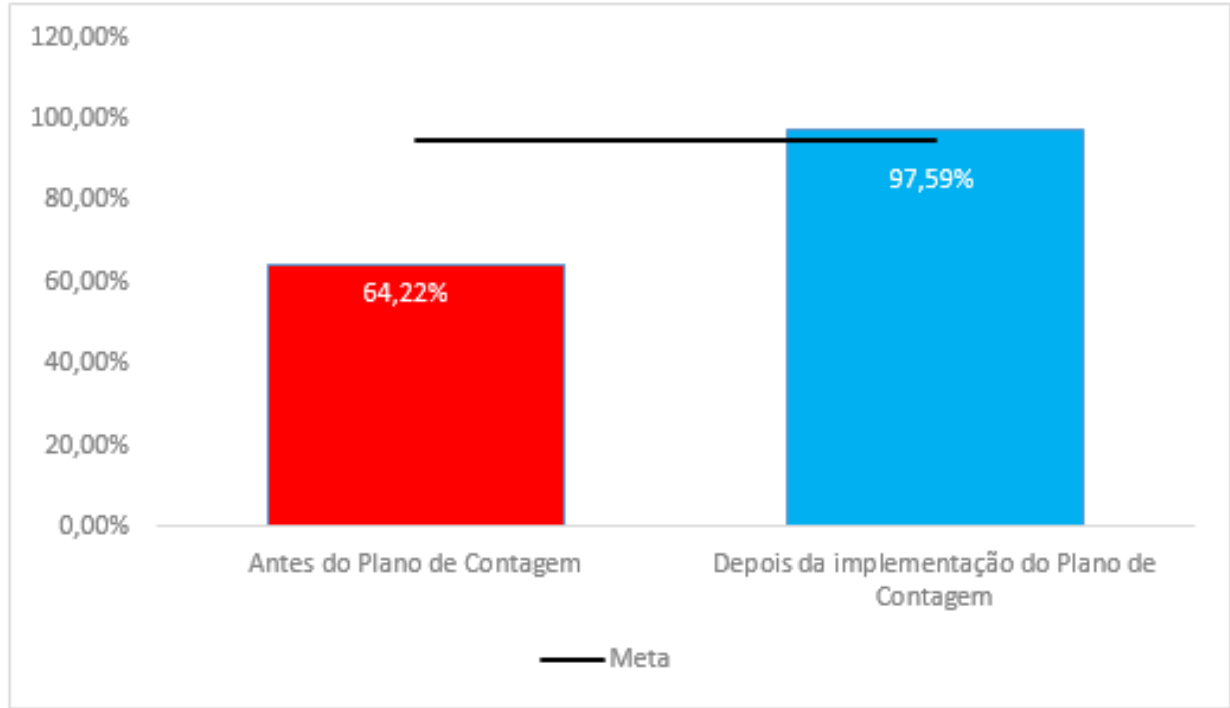

Fonte: Autores (2020)

A Figura 4, representam o resultado de inventário da classe A, antes e após o plano de contagem. Essa classe foi responsável, conforme Tabela 1, por $79,41 \%$ do valor em estoque no ano de 2019.

Pode-se observar que antes do plano de contagem, cerca de 87,5\% dos itens da classe A, ou seja, 07 itens apresentavam um índice de acuracidade fora dos padrões estabelecidos pela empresa e após o plano de contagem é possível verificar que $100 \%$ dos itens apresentava $100 \%$ de acuracidade.

Figura 4. Resultado do inventário dos itens da classe A antes do plano de contagem (a esquerda) e após o plano (a direita)
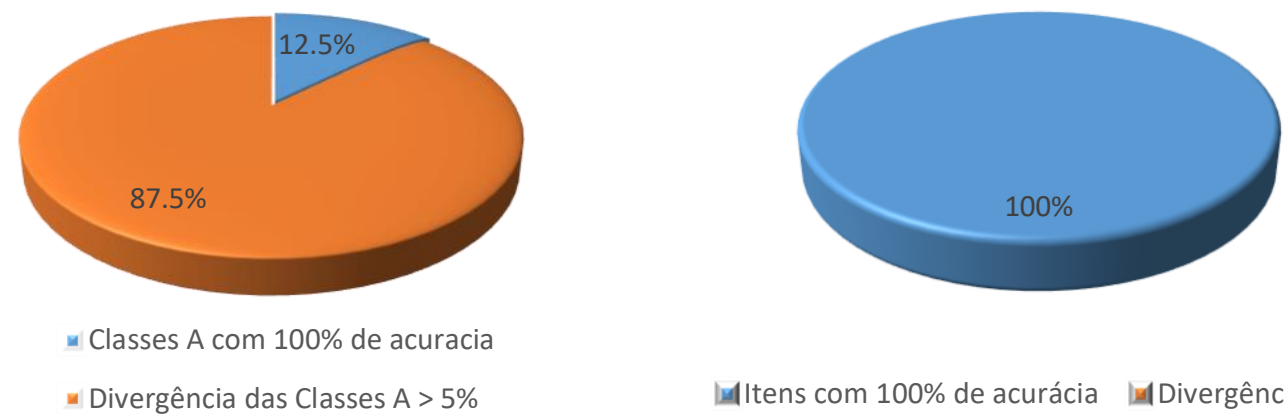

Divergência das Classes A > 5\%

國Itens com 100\% de acurácia 回Divergência > 5\%

Fonte: Autores (2020)

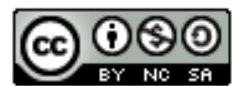


Citação (APA): Pereira, L. C., Fabbis, B. F., Oliveira, M. C. F. de, Oliveira, P. W. S. de, \& Abreu, S. R. de. (2020). Aplicação da curva abc e seu efeito na acuracidade do estoque em uma empresa de revenda de kit de embreagem na região da zona da mata mineira. Brazilian Journal of Production Engineering, 6(7), 58-67.

A Figura 5, representa o resultado do inventário dos 44 itens, antes e após o plano de contagem respectivamente. Pode-se observar que antes do plano de contagem, cerca de $72,73 \%$ dos itens de estoque, ou seja, 32 itens apresentam um índice de acuracidade fora dos padrões estabelecidos pela empresa e apenas $27,27 \%$, ou seja, 12 itens apresentam $100 \%$ de acuracidade.

Após o plano de contagem, pode-se observar que 95,45\% dos itens, ou seja, 42 itens apresentaram $100 \%$ de acuracidade e apenas $4,55 \%$ dos itens, ou seja, 02 itens apresentaram um índice de acuracidade fora dos padrões estabelecidos pela empresa.

Figura 5. Resultado do inventário antes do plano de contagem (a esquerda) e após o plano (a direita)

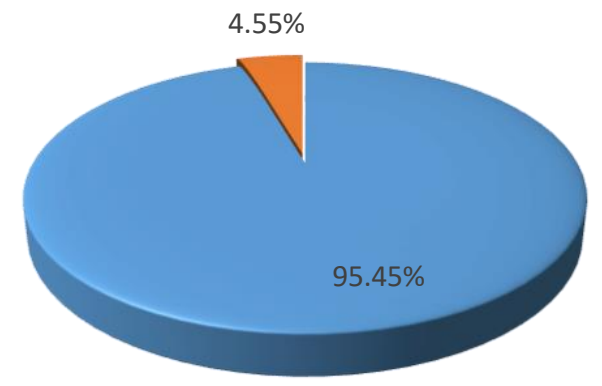

- Itens com $100 \%$ de acurácia uivergência > 5\%

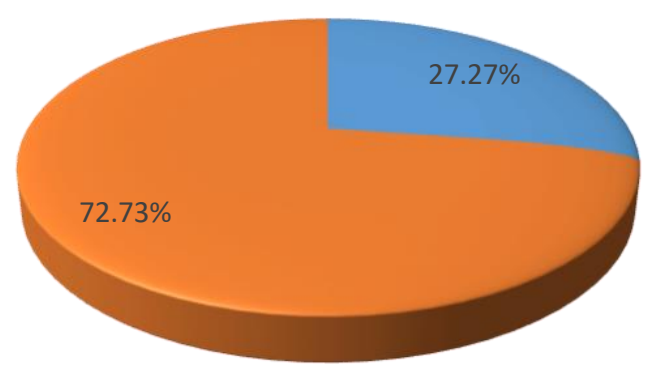

u Itens com $100 \%$ de acurácia - Divergência > 5\%

\section{CONCLUSÃO}

A curva $\mathrm{ABC}$ tem se mostrado uma ferramenta essencial no gerenciamento dos estoques, permitindo identificar a importância de cada item em relação ao custo total dos estoques, contribuindo assim para o uso de políticas de estocagem para cada classe de itens, dependendo da sua criticidade.

Ao aplicar a curva ABC na empresa em análise, foi possível identificar a criticidade dos itens que compõe o estoque e determinar uma melhor acurácia através do plano de contagem, proposto por Gasnier, utilizando-se um modelo de inventário cíclico, baseado na classificação dos itens.

Foi possível elevar a acurácia total considerando-se todas as classes, que foi elevada de $64,22 \%$ para 97,59\% em 3 meses de inventário cíclico. Considerando exclusivamente a classe A, podese perceber que antes da utilização do plano de contagem havia uma acurácia de $87,5 \%$ dos itens, não atendendo aos $95 \%$ estabelecido pela empresa; após o plano de contagem, $100 \%$ dos itens apresentaram acurácia de $100 \%$ e também que após o plano de contagem 42 itens apresentavam $100 \%$ de acuracidade.

O estudo demonstrou que a análise de estoque com o método da curva $\mathrm{ABC}$ tem efeito direto e positivo sobre o Índice de Acuracidade dos Estoques (IAE), porque auxilia na estruturação do plano de contagem e na otimização das tomadas de decisões. Com o uso da Curva ABC foi possível determinar um plano de contagem cíclico, de acordo com as criticidades de cada classe, identificando o tratamento que deve ser dado a cada uma.

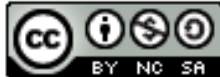



of Production Engineering, 6(7), 58-67.

Sugere-se que a empresa modernize o processo de requisição e baixa dos produtos do estoque através de tecnologia como uso de código de barras, inventário semestral ou quadrimestral e endereçamento de produtos.

\section{REFERENCIAS BIBLIOGRÁFICAS}

Accioly, F., Ayres, A. de P. S., \& Sucupira, C. (2015). Gestão de Estoques. Rio de Janeiro: Ed FGV.

Corrêa, H. L., \& Corrêa, C. A. (2017). Administração de Produção e Operações. Manufatura e Serviços: uma abordagem estratégica. São Paulo. Ed Atlas.

Facchini, E., Da Silva, J. R., \& Leite, V. M. (2019). Curva ABC e Estoque de Segurança. South American Development Society Journal, 5, 73. Disponível em: http://www.sadsj.org/index.php/revista/article/view/191. Acesso em: 03 mar. 2020.

Gerring, J. (2019). Pesquisa de estudo de caso: princípios e práticas. Petrópolis: Ed Vozez ltda.

Gomes, R. P., \& Milan, W. W. (2017). Gestão de estoque pelo método do estoque máximomínimo em uma empresa de médio porte do ramo de revenda de combustível em CarneirinhoMG. Organizações $e$ Sociedade. Disponível em: http://revista.facfama.edu.br/index.php/ROS/article/view/269. Acesso em: 03 de mar. 2020.

Hashimoto, M.; Candido, B. (2019). Empreendedorismo: Plano de negócio em 40 lições. São Paulo: Ed Saraiva Educação S.A.

Moreira, B. M., Silva, N. F., \& Ebias, D. G. (2019). Aplicação das curvas PQR e ABC como base para o desenvolvimento da estratégia de gestão de estoques em uma indústria farmacêutica do centro-oeste mineiro. Disponível em: http://aprepro.org.br/conbrepro/2019/anais/arquivos/10202019_221034_5dad081ee3400.pdf. Acesso em: 04 de mar. 2020.

Paoleschi, B. (2019). Almoxarifado e Gestão de Estoques. São Paulo: Ed Saraiva Educação S.A.

Ribeiro, J. (2017). Pesquisa de Marketing. São Paulo: Ed Senac.

Silva, B. W. (2019). Gestão de Estoques: Planejamento, Execução e Controle. João Monlevade: Ed BWS Consultoria.

Sousa, S., S. e S., E., Santos, G., R., S., \& dos Santos, T. (2018). Gestão de estoque em uma microempresa de atacado e varejo no município de Marabá-PA. Produção Em Foco. Disponível em: https://doi.org/10.14521/P2237-5163.2018.0017.0002. Acesso em: 03 de mar. 2020. 